\title{
Collaborative and Cognitive Network Platforms: Vision and Research Challenges
}

\author{
Ertan Onur • Yunus Durmus • \\ Mohamed Gamal Hawas • Sonia Marcela Heemstra de Groot • \\ Ignas G. M. M. Niemegeers
}

Published online: 9 April 2011

(C) The Author(s) 2011. This article is published with open access at Springerlink.com

\begin{abstract}
In this paper, we present a visionary concept referred to as Collaborative and Cognitive Network Platforms (CCNPs) as a future-proof solution for creating a dependable, self-organizing and self-managing communication substrate for effective ICT solutions to societal problems. CCNP creates a cooperative communication platform to support critical services across a range of business sectors. CCNP is based on the personal network (PN) technology which is an inherently cooperative environment prototyped in the Dutch Freeband PNP2008 and the European Union IST MAGNET projects. In CCNP, the cognitive control plane strives to exploit the resources to better satisfy the requirements of networked applications. CCNP facilitates collaboration inherently. Through cognition in the cognitive control plane, CCNP becomes a self-managed substrate. The self-managed substrate, in this paper, is defined as cognitive and collaborative middleware on which future applications run without user intervention. Endemic sensor networks may be incorporated into the CCNP concept to feed its cognitive control plane. In this paper, we present the CCNP concept and discuss the research challenges related to collaboration and cognition.
\end{abstract}

Parts of this work are presented in GLOBECOM, 2009 and 21st WWRF Meeting, 2008. This work is supported by the Trans Sector Research Academy for Complex Networks and Services, Delft University of Technology, the Netherlands.

E. Onur $(\varangle)$ · Y. Durmus · M. G. Hawas · S. M. Heemstra de Groot · I. G. M. M. Niemegeers Delft University of Technology, Mekelweg 4, 2628 CD Delft, The Netherlands

e-mail: e.onur@tudelft.nl

Y. Durmus

e-mail: y.durmus@tudelft.nl

M. G. Hawas

e-mail: M.M.G.E.Hawas@tudelft.nl

S. M. Heemstra de Groot

e-mail: S.M.HeemstradeGroot@tudelft.nl

I. G. M. M. Niemegeers

e-mail: I.G.M.M.Niemegeers@ tudelft.nl 
Keywords Cognition - Collaboration - Personal networks - Knowledge-based control · Cognitive control $\cdot$ Cooperative communications · Wake-up receivers

\section{Introduction}

The functioning of our society is becoming increasingly vulnerable to severe disturbances by natural (or man-made) causes such as large fires. The effects on people can be devastating and the economic loss may be high. There is an urgent need for tools to prevent, deal with and mitigate the effects of such severe disturbances. An example tool is the public safety services based on the cooperation of a large number of heterogeneous devices belonging to all potential public-safety actors operating in harsh wireless communication environments. The goal of such services is to prevent and deal with the public safety threats and emergencies where present communication systems are not up to the challenge. A dependable communication infrastructure is needed to allow effective collaboration and cooperation of all actors, ranging from different types of professionals (police, firemen, etc.) to the general public. Furthermore, citizens of the developed countries are becoming a source of sensing and image information through their personal devices, providing additional resources to deal with exceptional situations.

Consider an emergency case, for instance, a devastating earthquake. During and after the earthquake, cellular networks are generally out of order due to two main reasons: (1) impaired or damaged based stations and backbone networks; or (2) the base stations cannot handle the call requests in emergency cases where almost everybody tries to make calls and dropped calls are redialled [1]. Under such circumstances, the managed networks may fail to provide services. However, the networked devices may collaborate with each other to form an alternative operational network which may be used, for instance, to identify the position of a person stuck under a collapsed building. An opportunistically formed ad hoc network in the incidence area may provide group communications [2] and cooperation among emergency teams and forces, and other actors involved. There are also many other networks and resources available, public and private, infrastructure-based and ad-hoc, with different characteristics and aimed at different applications, that can assist in supporting the instances that deal with emergencies. Along with the recent proliferation of the endemic sensor networks, the number of these resources is expected to become abundant in the near future, creating a huge redundancy which may be exploited to enhance the provided quality of service or experience. If suitable solutions for cooperation were available, all these heterogeneous networks and resources could collaborate to provide a dependable and flexible communication, sensing and acting substrate.

Next to public safety services, another epitome is the arena of eHealth applications. For example, consider that an elderly person, who lives independently, has a serious health problem. An eHealth service provided to the elderly person may detect an emergency situation, for instance, through the wearable sensors. The personal monitoring system of the elderly person then raises an alarm for the emergency intervention of paramedics. As soon as a paramedic arrives at the elderly person's house, his networked devices collaborate with the elderly person's devices which are opportunistic to the paramedic. Such emergencies may happen at inconvenient places and times. Instead of enumerating all emergency cases and designing an application for each one of the enumerated cases, an intelligent and opportunistic network formation may adapt itself to the circumstances.

The networked devices proliferate, as diverse applications are introduced. The Wireless World Research Forum (WWRF) predicts that there will be 1000 wireless devices per person 


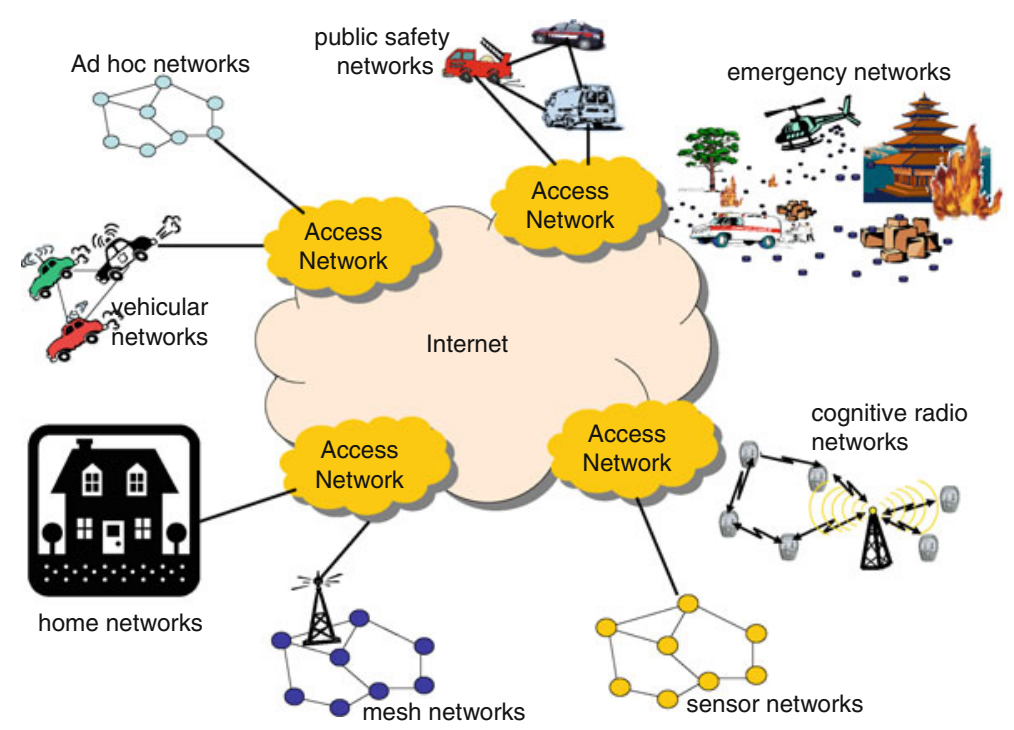

Fig. 1 A perspective on the present Internet

on average in 2017 [3]. When the population on earth is considered, the total number of nodes will be in the order of trillions. Designing a scalable architecture for such large networks, which may have to be formed opportunistically, will be a serious challenge. Beyond the scalability issues, management and control of the network will be very cumbersome if legacy solutions are employed in the design. Trillions of devices suggest more than a self-organizing architecture. The complexity of emerging ICT paradigms grows rapidly and the new technologies are heterogeneous, distributed and dynamic in nature as can be seen in Fig. 1. It is not realistic to assume management by human experts. We envisage Collaborative and Cognitive Network Platform (CCNP) as a new architecture which addresses these challenges and exploits cooperation opportunities in a cognitive way based on Personal Network technology [4]. CCNP is not an ad-hoc solution, tailored to a specific problem, but one that is based on an approach leading to solutions that are future-proof. It should be able to incorporate future developments in basic technologies, e.g., new radio interfaces, and be able to cope with evolving user requirements and objectives.

When the technology, experience and business drivers as presented in [5] are considered, CCNP provides a holistic architectural choice as depicted in Fig. 2. Technology started in the form of manual processes in history. Scientists recognized that adaptive systems may perform better than the automation which had replaced the manual operation. The technology trend is towards cognitive systems. In terms of business drivers, instead of stove-piped and isolated applications, transsectoral applications are envisaged where different business sectors cooperate with each other. CCNP will provide a substrate for transsectoral applications in which actors from multiple sectors closely collaborate and as such gain significant added value at reduced cost. Presently, business sectors develop their own communication infrastructure and solutions. This approach leads to non-scalable solutions when used in other business sectors. For example, voice over IP, may be considered as an initial transsectoral application. With the recent proliferation of communication technologies, users require a seamless architecture that alleviates the difficulties of system configuration and enhances usability. All these trends 


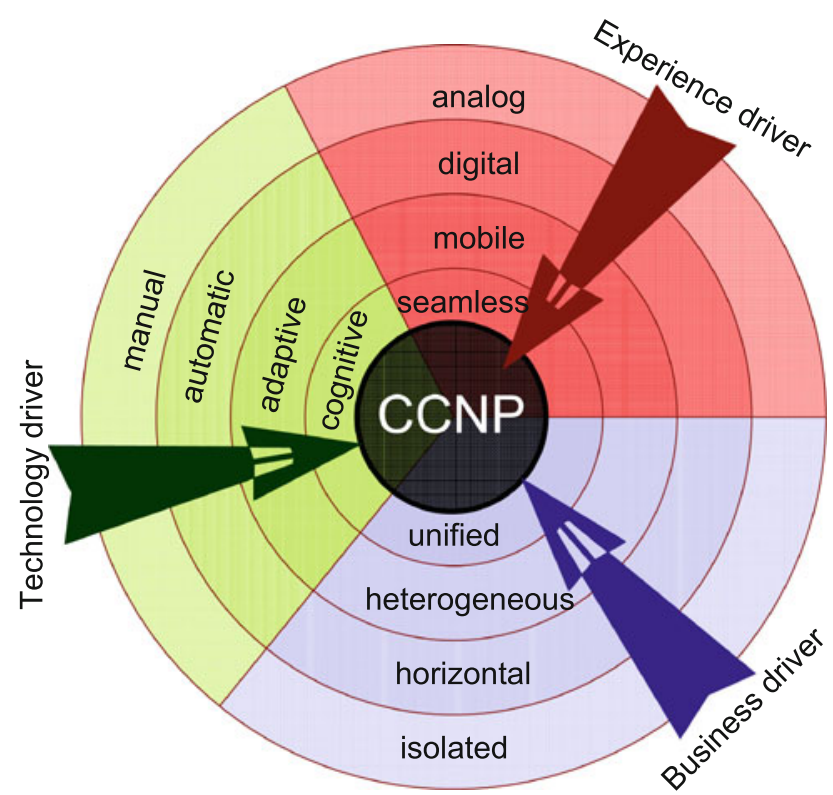

Fig. 2 Experience, technology and business drivers for CCNP. Redrawn from [5]

converge at the CCNP core which provides a unified, seamless and cognitive architecture facilitating collaboration as illustrated in Fig. 2.

The Present Internet evolved largely into a non-optimized static network. The limitations and the problems of wireless networking such as mobility of users, heterogeneity of devices/networks and interdependent security issues are not addressed adequately in the present Internet. The static environment created by the Internet is invalidated by the developments in mobile wireless communications. Sporadic disconnections, unreliable links and high error rates in wireless communication among heterogeneous power-limited devices stimulate research in the design of the Future Internet. Furthermore, recent developments in wireless sensor networks, in-body or body area networks, personal area networks, and delay tolerant networks must be incorporated in the Future Internet. Such networks create an explosion at the edges of the Internet [6]. One should not forget that the Internet of Things is emerging from the proliferation of cheap wireless devices and the opportunity to interconnect any artifact.

The present network architecture designs are based on known applications and user requirements considering the capabilities of the underlying networks, where network resource allocations are based on worst case scenarios. However, the requirements of future ad hoc applications and services are not known. Instead of tailoring applications based on the constraints of the communication technologies at design-time, an adaptive network may tailor itself and allocate resources dynamically on the fly, based on the application requirements and resource opportunities. With this scheme, applications development can be easier and faster. Furthermore, the applications can be portable across heterogeneous platforms with the help of virtualization technologies. An adequate solution is to make the network intelligent to make decisions and allocate resources opportunistically in the presence of information which may be: 
- incomplete: due to the large scale of the network, decisions may have to be taken based on local information.

- misleading or inconsistent: due to errors and dynamics in the networks, some pieces may already be invalidated at processing time. The operational conditions may change drastically and instantaneously.

- malicious: depending on the presence of malicious users, the produced data may also be misleading.

- highly correlated: due to the spatial-temporal dynamics of the network, the collected data may be redundant and highly correlated. An example may be the correlation of temperature measurements in a field by sensor networks or target presence decisions in surveillance applications [7].

Besides the unknown future application requirements, it is clear that the demand for larger throughput will continue to increase, driven by bandwidth demanding applications. Moreover, the number of networked devices will increase and require a larger aggregated bandwidth of the order of Tbits per second. Another significant issue in the deployment of future networks will be the large bandwidth requirement associated with the transportation of the application data.

The threats to the security and safety of humans change continually in time. For instance, a PEW Internet survey shows that $66 \%$ of the experts contributing to the survey expects a devastating attack on the networked information systems in this decade [8]. Internet, being a scale-free network, is robust against random errors. However, due to clustering and having nodes with a large degree, Internet becomes highly susceptible to intentional attacks to the highly-connected routers [9]. Decentralizing network architectures is a counter measure for such attacks. Furthermore, networks must be resilient to attacks by being adaptable to such exceptional circumstances [10]. The measures implemented up to now could not yet manage to solve the problems adequately. Sagarin et al. conducted cross-disciplinary research to determine the common patterns of dependability in natural systems. They assert that the best place to search for a dependable solution is nature. Many organisms have managed to survive for millions of years and defeated the encountered threats by decentralization, adaptation and cooperation [10]. Hence, we claim that cognition for adaptation and cooperation may enhance the security of the Future Internet which will natively be a distributed environment. CCNP may also enhance the dependability of the Future Internet from this perspective.

CCNP is a distributed platform which employs cognition to enhance the adaptation capabilities of its entities and enables cooperation inherently. The cognitive aspect at the core of CCNP goes beyond cognitive radio. In this paper, we present the CCNP concept and the associated research challenges. We present the challenges in two domains: collaboration and cognition. These two domains may appear as orthogonal concepts to each other. However, there is interdependence. Cognition may facilitate collaboration and vice versa. The interdependence will become clear throughout the text.

This paper is organized as follows. In Sect. 2, the CCNP concept is presented. In Sects. 3 and 4, the research challenges related to collaboration and cognition are discussed, respectively.

\section{Collaborative and Cognitive Network Platforms (CCNP)}

Cognitive networking [11] aims at introducing intelligence into the network architectures that goes beyond simple adaptation capabilities. Collaborative and Cognitive Network Platforms 


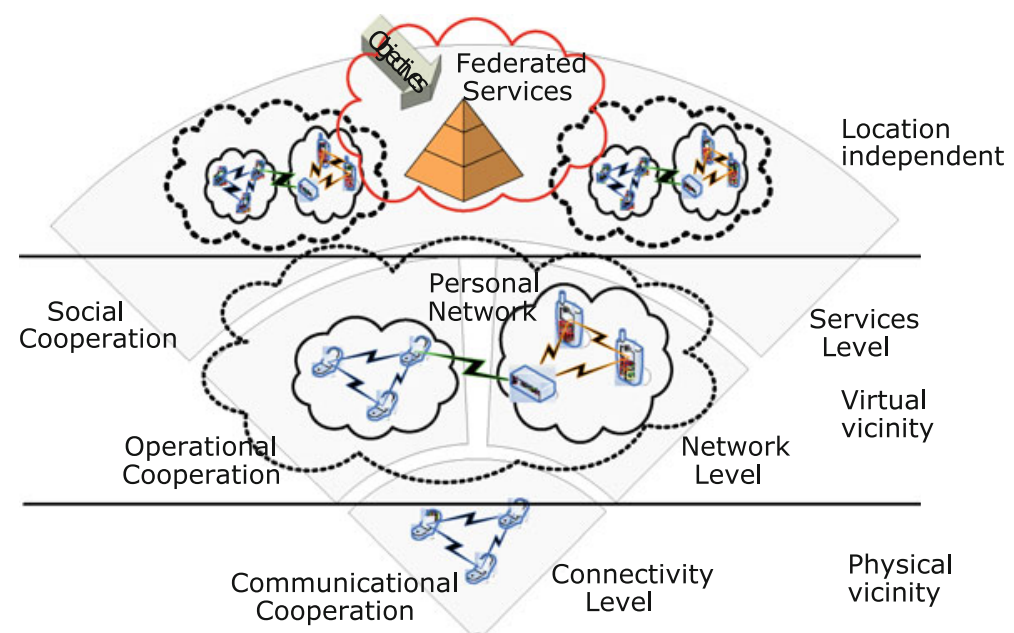

Fig. 3 Personals Networks may facilitate cooperation at different levels

(CCNP) is a visionary concept, derived from Personal Networks (PN). PNs are distributed cooperative systems which provide an inherently secure and cooperative distributed environment for personal devices. They are self-organizing systems that adapt automatically to spatial-temporal dynamics such as availability of resources [4].

A PN autonomously interconnects the personal devices of the user in a secure fashion and offers users access to the services provided on the nodes. Users access and use services through any device in the PN. A personal area network connects the devices located in the vicinity of each other, whereas, PNs provide virtual vicinity for geographically distributed devices. In a PN, nodes may communicate with each other independent of their location as shown in Fig. 3. At the connectivity level, various heterogeneous technologies form clusters in a self-organized fashion in order to exploit the scarce resources better. At this level, the communication takes place in the physical vicinity. By abstracting the connectivity level using the Internet Protocol (IP), the Network Level enables cooperation among distributed clusters by forming a self-organized overlay network. At the services level which resides on this overlay network, services, applications and users may collaborate with each other. The overlay network facilitates collaboration independent of the location of the devices.

Such overlay networks may federate with each other to form larger collaborative systems. A PN federation (PNF) is defined as a temporal, ad hoc, opportunity- or purpose-driven secure network of independent PNs. Example scenarios of PNFs include project networks, where colleagues organize a project meeting and federate their PNs to prepare project documents and health-care networks, where family members and medical professionals federate to allow access to medical sensor information of patients. By federating, some subset of personal resources or services is made accessible to other people to achieve a common goal.

PNs and their federations provide a natural basis for introducing cognition into all system layers to achieve particular system objectives. In CCNP, the cognitive control plane may be deemed as the entity where cognition is introduced into the architecture as shown in Fig. 4. Sensor networks and other architectural components provide context information about the environment, user and applications to the cognitive control plane. The context information is then collocated and analyzed to predict the user preferences and conditions of the entities of the communication and computation substrate. Gathered history information is used 
Fig. 4 Control plane in the collaborative and cognitive network platforms

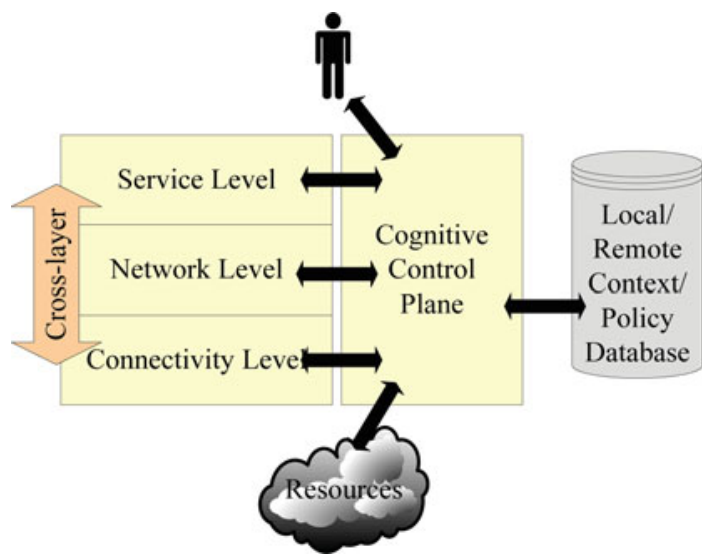

to determine the patterns, to produce decisions at several protocol layers and to fine-tune the components. In this way, objectives and requirements of applications and users can be satisfied and conflicts among the objectives may be solved with minimal user intervention.

The cognitive control plane provides smart decisions to control the resource sharing operations, and collects and manages the state transitions over the lifetime of resource sharing operations. In CCNP, the decisions are produced by the cognitive control plane as shown in Fig. 4. CCNP provides a natural basis for introducing cognition into all system layers to achieve particular system objectives. The goal of the cognitive control plane is to achieve a network architecture capable of redesigning itself or changing its strategies according to a set of objectives such us improving the quality of service or optimizing the management of the available resources. Since the cognitive engine has to solve multi-objective optimization problems, the solutions are Pareto-optimal [11]. Based on the gathered context about the resources, user objectives and mobility patterns, the cognitive engine plans, decides and executes the proper action such as adapting a protocol parameter or allocating physical resources to applications or services. The elements in the cognitive engine may be neural networks, swarm intelligence, evolutionary programming, immune or fuzzy systems, etc. The cognitive engine adapts itself continuously by using the context database, and a priori actions and their feedbacks.

When the heterogeneity of the underlying devices and networks are considered, there will be a large number of resources. These resources must be represented as virtual resources and mapped to physical resources dynamically to enable portability of applications and to enhance the resource utilization without affecting the already developed applications. Therefore, one of the research challenges is mapping of the resources to corresponding virtual resources [6]. The cognitive control plane does the mapping on the run. This sort of mapping must provide enough information about the capabilities of the resources, while the device/network capability information must be harmonized in the CCNP. In this way, CCNP can be considered as a distributed middleware abstracting all kinds of heterogeneity in the physical resources as shown in Fig. 5.

The cognitive control plane may identify faults based on the information obtained and stored in a context and state database. While filtering out useless information, the states of the virtual and physical resources and their run-time mappings may be monitored and fault tolerance decisions may be fed back to the cognitive control plane. On identification of the faults, context switching and other fault management decisions and actions may be suggested to the communication stack. Furthermore, the cognitive control plane may facilitate 


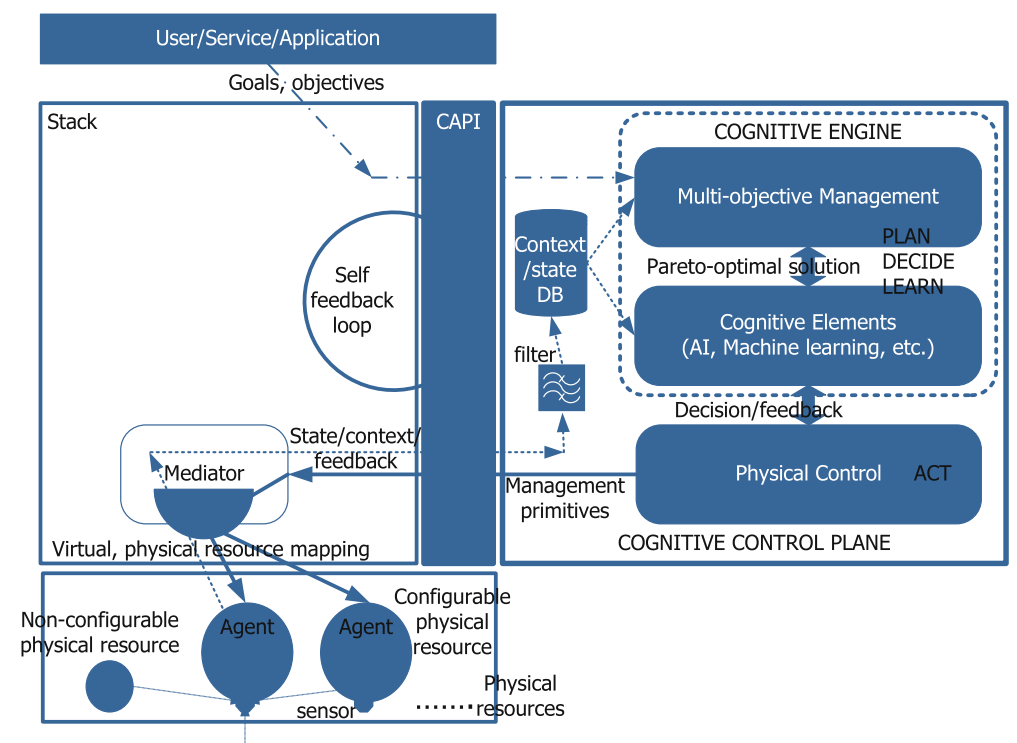

Fig. 5 Control plane in the collaborative cognitive network platforms

exploitation of the redundancy in the physical resource pool. For instance, the cognitive control plane may take decisions as to where the content should be stored and replicated.

The optimization decision taken by the cognitive engine must generally be produced rapidly on the fly. Various techniques can be adopted, however, learning from the past-experiences and producing a policy set would be the most rapid approach. Matching the current state to a clustered policy base, will enable producing decisions faster. Therefore, the cognitive control plane runs some cognitive elements in an offline fashion to produce and update the policy database periodically or when a significant event that may change the decisions happens in an event-driven way. Then, when a decision is to be produced, pattern classification techniques can be applied using some similarity measures to match the observed state to a policy which in turn can be used to derive the actions.

The CCNP requires input through observations of the states of the virtual or physical resources; e.g., state of the congestion levels in the network, to which the multimedia trunks are assigned, residual battery levels, queue sizes of the services, memory utilization of the applications or user behaviors. Although we give examples related to networking applications, the CCNP concept is a generic concept which can be extended to other domains. To learn the behavior of the infrastructure, context information must be gathered and stored in a database. Wireless sensor networks or other networks and elements can be employed to gather the context. The context is not only related to the physical entities in the system, but also to information about the CCNP entities, services, applications and also users. Instead of using the vast amount of unprocessed data, the input can be filtered to extract the useful information that can be considered as feature extraction. Producing accurate and timely decisions and the price of the ignorance caused by extraction of the data considered to be irrelevant are some of the research questions.

A cognitive element that can be employed in the cognitive control plane for multi-objective resource optimization is machine learning. It is in charge of maintaining experience of resource utilizations. The resource utilization knowledge must be consolidated and invalidated 
in the context/state database. Machine learning can be employed as a classification learning tool. The classification is a function that maps the states to a (or a set of) strategies. The cognitive control plane needs to enrich the context/state and policy database continuously by introducing new strategies and policies. Inductive logic programming is suitable for this task.

After briefly introducing the CCNP concept, we present the two seemingly distinct concepts, collaboration and cognition in Sect. 3 and 4, respectively.

\section{Collaboration}

In a collaborative system, independent individual components work together to create a value greater than the sum of the values of the individual components. This is a common natural process that can be seen in life; e.g., cells form organs which form humans which form societies. Distributed wireless networks facilitate cooperation at all levels of the systems starting from the physical layer to the applications and enable cooperation among users. For example, cooperative relays increase the throughput; multi-hopping intensifies the connectivity; transsectoral services facilitate re-usability and cross-layer designs enhance the efficiency.

For example, consider an ad hoc network of nodes where a reactive routing protocol is employed. When a node has a packet, the routes are discovered. Assume that there is a malicious or selfish user in the network which does not contribute to the route discovery process by relaying the route request packets. If the user is a malicious one, in this way, some part of the topology can be hidden. If the user is selfish, it may drop the route request messages to conserve its energy. For both cases, the consequence can be detrimental depending on the topology especially when the malicious user is acting as the only bridge between two sub-networks. In this case, two-sub-networks are disconnected depending on the operation of the malicious user. The other nodes cannot individually determine the existence of a malicious user. A rating mechanism coupled with cooperation (interaction among the nodes to exchange the ratings of neighboring nodes) is required to identify the malicious or selfish users. Such defectors may exist in any kind of social or physical network.

Natural selection may favor for defectors rather than the cooperators in unstructured populations [12]. However, cooperation is one of the fundamentals of the evolution of biological systems. Ohtsuki et al. present a very simple rule for the case in which natural selection favors cooperation. They claim that if the ratio of the benefit, $b$ to the cost, $c$ of an act exceeds the connectivity degree of the individual, $k$ (i.e., if $b / c>k$ ), cooperators dominate defectors. A cooperator is defined to be the individual that pays a cost $c$ to receive a benefit, $b$ and a defector pays no cost and does not provide benefits to others [12].

Repeated interactions among the nodes may increase the level of cooperation. If players meet for the first time in the prisoner's dilemma, the optimal option is to defect. However, as the number of interactions increases, cooperation may be favored. Beyond the successful titfor-tat strategy (cooperate initially and continue to cooperate as long as the partner cooperates in the previous stages), raise the stake strategy is also another one which is about being nice, retaliating and forgiving [13]. In the raise-the-stake approach, individuals invest a low stake in their first encounters with each other and they raise the investment with the cooperators in a reciprocal fashion. This strategy minimizes the losses at the first encounter. Consequently, a trust relationship is built progressively as the individuals interact with each other. This fact suggests that there is a correlation between cooperation level and friendship measured in terms of connectivity [13]. Interactions impact the level of cooperation [14]. Interactions may be local if the population has a spatial structure. In CCNP, this phenomena can be explained as cooperation at the connectivity level within the physical vicinity. Interactions can also be 
remote which requires some information exchange. When remote interactions are needed, the cooperation strategy may be implemented at the network or services levels as shown in Fig. 3.

It is shown that animals and humans cooperate much more than the level predicted by the present game theory [15]. Mike M.-Gibbons discusses in [15] that a new kind of game theory must be developed to understand the impact of several other factors such as group dynamics or network structures by considering the players of the game as complex adaptive systems.

In the sequel, we briefly elaborate on the collaboration techniques that are being employed in the present networking technologies. The collaboration techniques being employed in networks are categorized as vertical, horizontal and dual-stack collaboration. In the vertical collaboration, different layers in the ISO/OSI model share information with each other to satisfy the requirements. Peer layers in the communication stack cooperate with each other in the horizontal dimension. If there are multiple stacks in the communication architecture, e.g., wake-up radio in sensor nodes, then the distinct stacks collaborate with each other in the multiple-stack approach.

\subsection{Vertical Collaboration}

A recent problem statement to IETF clearly describes the vertical collaboration requirement [16]. In this Internet Draft, it is stated that applications and services cannot efficiently use the provided network capabilities due to the lack of interaction between the networked applications and the network [16]. This deficiency leads to poor exploitation of the resources and degradation of the quality of service. Cross-layer interactions, which we refer to as vertical collaboration in this paper, are required. For example, medium access control (MAC) protocols or link-state based routing algorithms may leverage the awareness in the dynamics of the radio channel.

The ISO/OSI layers abstract the communication and networking functionalities that may easily become convoluted. Although abstractions enable modular designs, they also introduce additional challenges. An essential difference between wired and wireless networks arise especially when quality of service provisioning is considered. For example, assume that TCP is used at the transport layer in a wireless network. Providing quality of service at the transport layer requires differentiating congestion in the network from bit errors in the physical layers, packet errors (e.g., ACK) due to collisions at the data link layer or from packet error due to routing failures (e.g., in a mobile ad hoc network). Tackling such problems requires cross-layer interactions and collaborations. The performance of a system may be enhanced by vertical collaboration which is enabled with the interactions among layers in a stove-piped system. Vertical collaboration is generally facilitated through cross-layer implementations. For instance, link state routing protocols developed for ad hoc networks use information from physical and data link layers. Advanced cross-layer designs such as knowledge-plane are discussed in [5] by Fortuna and Mohorcic. Some simple examples of vertical collaboration are presented in Table 1. Vertical collaboration raises the cooperation level in an entity; however, horizontal collaboration is required for improving the performance of the system and entity in their interactions with other entities or with each other.

\subsection{Horizontal Collaboration}

There are various cooperation techniques between peer layer when the ISO/OSI layering architecture is considered. At the physical layer, cooperation may be achieved by transmitting 
Table 1 Examples of horizontal collaboration opportunities at different ISO/OSI layers

\begin{tabular}{|c|c|c|c|c|c|}
\hline \multirow[t]{2}{*}{ Example } & \multicolumn{5}{|c|}{ Layers } \\
\hline & PHY & MAC & NET & TRP & APPL \\
\hline Transmission adaptation [17] & $\checkmark$ & $\checkmark$ & & & \\
\hline MAC scheduling [18] & $\checkmark$ & $\checkmark$ & & & \\
\hline Link state routing [19] & $\checkmark$ & $\checkmark$ & $\checkmark$ & & \\
\hline TCP over wireless channels [20] & $\checkmark$ & $\checkmark$ & $\checkmark$ & $\checkmark$ & \\
\hline File distribution systems [16] & $\checkmark$ & $\checkmark$ & $\checkmark$ & $\checkmark$ & $\checkmark$ \\
\hline Streaming content distribution systems [16] & $\checkmark$ & $\checkmark$ & $\checkmark$ & $\checkmark$ & $\checkmark$ \\
\hline
\end{tabular}

PHY physical layer, MAC medium access sublayer of data link layer, NET network layer, TRP transport layer, $A P P L$ application layer

the same signal by multiple nodes in the network to reduce the bit error rate or to conserve energy. When the medium access protocols are analyzed, the nodes may cooperate to resolve the channel access conflicts and collisions. At the network layer, relaying is one of the simplest cooperation technique. At the transport layer, nodes on a route may cooperate to reserve bandwidth in order to provide quality of service to the applications. In general, the peer layers of the protocol stack cooperate with each other to satisfy the requirements in the horizontal collaboration. Some examples of the horizontal collaboration techniques are presented in Table 2. In the following sections, we elaborate on some of the examples shown in Table 2.

\subsubsection{Physical Layer}

Wireless networks, thanks to their distributed nature, facilitate collaboration and distributed signal processing which may help conserve energy significantly and increase efficiency. Wireless services require a certain quality of service (QoS) for reliable operation. Data and video communication systems require a higher degree of QoS compared to voice communication systems. QoS may be measured in terms of the bit error rate (BER). For example, the BER requirement for WiFi (wireless local area networks) and GSM (Wireless mobile telephony) are $10^{-5}$ and $10^{-3}$, respectively. As more and more ICT-enabled energy conservation systems rely on wireless communications, those systems pose serious environmental concerns themselves. Therefore, energy preservation by employing smart signal processing and communication techniques is of paramount importance. One way to increase power efficiency of such communication systems is to employ multiple antennas. This is easier to achieve in wireless access points or base stations than in portable mobile terminals due to the size constraints. Consequently, a need is felt for other power efficient techniques. Cooperative communications is a key enabler to boost the performance of wireless communication links and offers significant power savings. The cooperation strategy is to transmit the same signal in a coordinated fashion by multiple terminals as can be seen in Fig. 6. Therefore, in a cooperative communication environment each mobile acts both as a user and a relay at the physical layer. This helps to counter the deleterious impact of fading which is typical of a wireless communication channel and helps to boost the system performance.

In cooperative communications (CC), nodes in a network (e.g., of a WiFi network) help each other communicate their signals over independent fading channels. For example, as can be seen in Fig. 6, User 2 helps User 1 to transmit the signals to the access point. In step 1, when User 1 broadcasts its message, the access point (AP) receives the signal. In the 
Table 2 Examples of vertical collaboration techniques and opportunities

\begin{tabular}{|c|c|}
\hline ISO/OSI layer & Examples \\
\hline Application layer & $\begin{array}{l}\text { Transsectoral services and applications } \\
\text { Context provisioning by sensor networks to applications } \\
\text { Rating of users for setting up trust relationships } \\
\text { Identification of malicious or selfish users } \\
\text { Femtocells facilitating cooperation with operators and end-users }\end{array}$ \\
\hline Transport layer & $\begin{array}{l}\text { Quality of service provisioning } \\
\text { Conflict resolution in service level agreements } \\
\text { TCP over wireless channels through proxies in splitting approach }\end{array}$ \\
\hline Network layer & $\begin{array}{l}\text { Relaying and routing } \\
\text { Multi-point relays for minimization of control traffic (e.g., OLSR) } \\
\text { Identification of stale routes } \\
\text { Route caching } \\
\text { Adaptive hybrid routing }\end{array}$ \\
\hline Data link layer & $\begin{array}{l}\text { Collision resolution } \\
\text { Rate adaptation using beacons } \\
\text { Transmission power adaptation } \\
\text { Wake-up receivers for channel reservation } \\
\text { Spectrum sensing using sensor networks for cognitive radio networks } \\
\text { Distributed localization }\end{array}$ \\
\hline Physical layer & $\begin{array}{l}\text { Cooperative communication } \\
\text { Network coding } \\
\text { Frequency reuse through cooperative communication } \\
\text { Cognitive radio to increase spectrum efficiency } \\
\text { Wake-up receivers for event-driven communications [21] }\end{array}$ \\
\hline
\end{tabular}

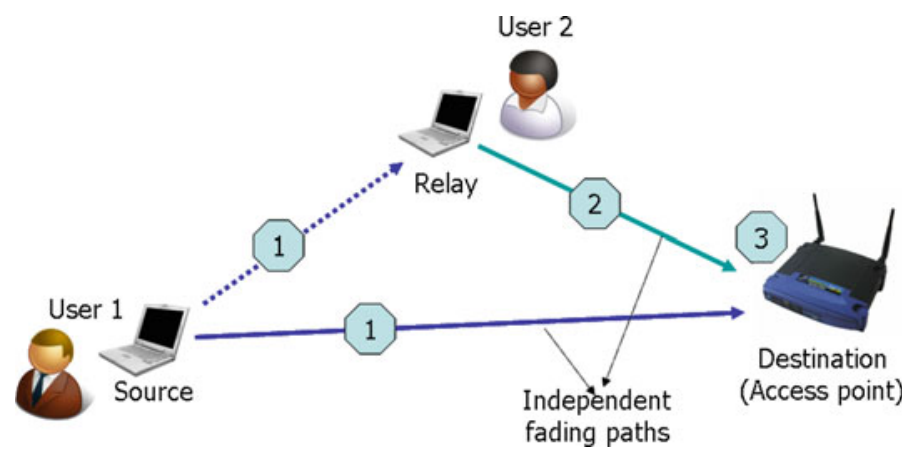

Fig. 6 Cooperative communications example

meantime, User 2 can also receive the same signal. Since relay (aka helper or cooperator) is closer to both source and destination, the signal-to-noise ratio (SNR) at the relay (node belongs to User 2) is larger compared to the SNR at the destination (AP). At step 2, the relay node sends the same signal to the AP. Then, when the AP receives both signals, it decodes the message by employing a signal combination technique (step 3 in Fig. 6). Since the same 
message is conveyed to the AP over independent fading channels, the SNR at the destination is larger and a lower BER can be achieved. Assume that a power budget is allocated to the source. Some portion of this budget can be employed for relaying (without any additional power requirement) and a lower BER can be provided at the destination when CC is employed compared to direct line-of-sight (LOS) communications. Instead of decreasing the $\mathrm{BER}$, it may be kept the same and then cooperative communications can facilitate reducing the overall energy consumption.

A number of investigations regarding the performance gains of cooperative communications in wireless networks have been carried out. Our recent study [22] reveals the impressive gains obtained by cooperative communications compared to a non-cooperative communication (NCC) system. Average received signal-to-noise ratio (SNR) required for achieving a BER of $10^{-3}$ in LOS and non-line-of-sight (NLOS) wireless communication scenarios for standard non cooperative communications (NCC) and cooperative communications are studied. By just employing a single cooperative relay, the source energy can be conserved up to $50 \%$ for the LOS case. For NLOS, the gains are event more impressive [22].

Cooperative communications can also increase the data rate. Consider a situation in which the direct path between the source and destination has a low bit rate compared to the sourceto-relay and relay-to-destination data rates. In this case, the total delay of indirect path is less than direct one. Other advantage can be the frequency reuse. The unlicensed frequency band is a scarce resource. So each frequency band must be reused in other cells. For instance, in a cellular network there is a pattern to allow frequency reuse. With help of cooperative communications and proper power allocation, the signal strength of a single path is much less than the traditional method. So the signal to interference ratio will decrease and this can help the system performance [22].

\subsubsection{Data Link Layer}

Collaboration at MAC layer is introduced in [23], where a source node selects a single partner upon link quality basis before initiation of data transmission in a proactive way. Collaborative relaying takes place when a transmission failure at the sender is detected. Only DATA and ACK frames are cooperatively relayed. RTS/CTS messages are disabled, they are enabled only when the sender experiences transmission failures with its partner. Cooperative Diversity MAC (CD-MAC) outperforms conventional MAC with respect to packet delivery ratio and end-to-end delay in a noisy environment. A cross layer coding approach is presented in [24] to improve BER performance and reduce the complexity of the receiver. The scheme is based on Hybrid Automatic Repeat Request (H-ARQ) and works in a cooperative relaying manner. Data encoding/decoding is carried out via a cross layer approach using both physical layer and upper layer forward error correction (FEC) scheme. The proposed mechanism has better BER and signal-to-noise ratio performances compared to conventional approaches.

\subsubsection{Network Layer}

Multi-hop communication and relaying is one of the basic collaboration techniques which has been employed for many years. We showed that a single relay device properly positioned in the middle of the network area at the same height with other nodes can reduce path loss by $33 \%$ [25]. This result motivates the multi-hop communication for $60 \mathrm{GHz}$ indoor networks and two hops are generally sufficient for typical indoor scenarios. Using relay nodes provides advantages on amount of path loss due to nonlinear relation between distance and path loss. 
The delay imposed by relaying degrades the network throughput by a factor of two. Whereas, the throughput is reduced by a factor of 10 in $60 \mathrm{GHz}$ networks due to an obstacle causing $10 \mathrm{~dB}$ attenuation [26]. It is possible to bypass an obstacle and keep connectivity of network via relay. Considering the distribution of network nodes in a $60 \mathrm{GHz}$ home network, two-hop communication is satisfactory for most of the cases [27]. One relay may sufficiently enhance the connectivity in $60 \mathrm{Ghz}$ networks. If the direct link between two nodes is blocked with an obstacle, the gain due to relaying will be larger since the direct link will suffer from severe attenuation. In these scenarios, no intelligence is assumed in the protocols. If there are intelligent devices which are capable of assessing the link quality, then nodes may select the best path among two options and larger gains may be achievable. $1.8 \mathrm{~dB}$ gain is the worst case gain provided by relaying among all possible cases [25].

\subsubsection{Application Layer}

Future networks will be composed of many heterogeneous networks. We envisage that services will be provided on such networks to and from different business sectors. This fact can already be observed in the fixed-mobile convergence of the voice over IP applications on the Internet. Furthermore, end-users (consumers) will generate and deliver services to other consumers. A generic infrastructure is required for supporting such heterogeneous services from dissimilar sectors. When other sectors such as eHealth and energy are considered, convergence is more challenging. This concept is referred to as transsectoral infrastructure [28]. Designing a generic substrate is the objective of transsectoral concept to sustain applications of different business sectors rather than employing stove-piped and dedicated applications and networks for each sector. The transsectoral infrastructure may then facilitate collaboration among diverse business sectors to enhance the quality of the lives of citizens by introducing an integral cooperative architecture formed of different sectors in the society (e.g., eHealth, education, energy). Individual sectors may be considered as nodes in a graph, and the edges may represent possible interactions and contributions. Contributions (skill, finance, product) from different sectors can provide durable solutions for existing problems and meet future social demands. We believe that CCNP may be the generic architecture aimed at by the transsectoral concept.

To achieve the transsectoral concept, heterogeneous technologies should converge to collaborate with each other. One of the adequate examples of the convergence in cellular networks is through the femtocells. Femtocells facilitate cooperation between the end-users and network operators by reducing the site management and energy costs of the operators. Femtocell is a small cellular base station designed to be used in residential or indoor environments $[29,30]$. This small device provides indoor wireless coverage and uses the existing broadband Internet connections for backhauling the traffic to the network operator. The need for indoor cellular coverage is becoming a necessity because $3 \mathrm{G}$ mobile technologies are spreading despite their poor indoor coverage. The femtocell concept is applicable to all kinds of cellular systems. The mobile network subscriber purchases the femtocell through the subscribed mobile operator, installs it and the operator activates the femtocell utilization. The subscriber, then, uses the legacy handset to communicate through the femtocells. To enable operation in the licensed spectrum, the operators need to manage the device and the femtocell must share the purchased spectrum with the macro cells. Although, currently femtocells are produced for existing cellular network standards, such as UMTS, products supporting WiMAX and LTE are emerging, as well. Femtocells can also be deemed as fixed mobile convergence gateways due to their support for dual mode operations. To exploit the femtocell opportunities, 
the Femto Forum was established as a not-for-profit membership organisation in 2007 [31] with the objective of developing open standards for product interoperability. The technical challenges of femtocells are listed in [29] as resource allocation, timing/synchronization, interference management, mobility support, bridging femtocells with operator network over IP and backhaul QoS.

\subsection{Multiple-Stack Collaboration}

Embedded systems, pervasive communications and wireless sensor networks have recently been considered as cooperating objects [32] with great synergetic potential in achieving various objectives, in particular in the realm of significantly reducing energy consumption. The basic idea is that individual entities (sensors, actuators, etc.) strive to reach a common goal by collaborating. Energy is the bottleneck of sustainability in many critical applications. For example, in a home automation system where energy consuming devices are controlled through cooperating objects, keeping all devices operational all the time results in excessive energy consumption. Instead of always-on or sleep-scheduled systems that have been proposed until now, smart event-triggered, on-demand operation of the network may improve the energy efficiency and the lifetime of the system.

For successful data delivery, the destination nodes should be listening to the medium to receive data when the sender node starts data communication which is referred as rendezvous [33]. The most energy-efficient rendezvous scheme is utilizing wake-up radios. Pure synchronous rendezvous requires recurrent time synchronization and consumes considerable energy because of idle listening or overhearing. In pseudo-asynchronous rendezvous, source nodes wake up and emit a preamble signal. The preamble time is long enough to coincide with the schedule of the destination node. Upon sensing the preamble, the destination recognizes the intended packet transmission. Time synchronization is not required, but sensors still follow a duty cycle and consume considerable energy with preamble signalling. In pure asynchronous rendezvous, sensors reside in deep sleep and can be woken up by their neighbors on demand with very low-power event-driven radio receivers. Whenever a node intends to send a packet, at first it wakes up the destination node, then sends the packet. Therefore, event-driven radio is a solution to the redundant energy consumption caused by rendezvous.

Though the concept of event-driven networking is known, an additional wake-up receiver may significantly change the energy efficiency of the communications systems by a cooperative dual PHY-MAC stack. In this approach, an event-driven stack is cooperating with the main communication stack to maximize the efficiency of the communication. A new crosslayer concept is brought up in this approach where a low-power radio enhances the operation of the main radio. Existing work mostly addresses the wake-up receiver design issues and does not consider cross-layer multi-hop networking issues. There are still open theoretical and practical problems. For example, event-triggered cooperative clustering techniques need to be developed to conserve energy through exploiting the spatiotemporal correlations among events [34]. The cooperative and cross-layer dual stack may adapt the network to the event rate and consequently conserve energy or help the main stack to mitigate various problems such as collision avoidance. Novel topology management techniques have to be developed to mitigate the impact of the irregular communication ranges of the wake-up and main radios. 


\section{Cognition}

The roots of cognition in computer science lie on Cognitive Psychology which was introduced by Ulric Neisser in 1967. According to Ulric Neisser "cognitive psychology refers to all processes by which the sensory input is transformed, reduced, elaborated, stored, recovered and used" [35]. This definition of Cognition states that the process begins with the collection of sensory input, continues with filtering, transforming the input and finishes after storing it as meaningful data. Later, the stored data is recalled and used in our actions.

In communication networks, the term Cognition was first used by Mitola in [36] as "the mix of declarative and procedural knowledge in a self-aware learning system." Mitola's definition of cognition depends on cognitive psychologists' knowledge descriptions. Declarative knowledge refers to information that is static and unchanging, whereas procedural knowledge tends to be dynamic and changing [37]. Procedural knowledge can be simply defined as the information that it is easier to show to someone than it is told, like describing the principles of riding a bicycle. Besides this definition of cognition, in cognitive networks it is mostly referred to as the cognition cycle [36] (feedback loop in [38]). Cognition cycle is a learning model where there is a feedback loop which elaborates and transforms the information according to the feedbacks gathered as a response to our actions. The actions that we perform, originate from the prior knowledge which are collected from outside, then reduced and elaborated after the feedback (learning phase). As mentioned in [38] Observe, Orient, Decide, Act (OODA) is an example of feedback model that is used in military processes.

\subsection{Proposed Architectures}

In [38], the cognitive process is designed to involve three components: the specification language, cognition layer, and network input. These components follow the feedback loop discussed above. The specification language is a kind of QoS specification language which aims to connect the requirements of the application layer to the cognitive layer. The cognition layer is where the cognition loop is placed. It can be set up as centralized or distributed according to the aspects of the network. Machine learning techniques such as artificial neural networks and genetic algorithms could be incorporated in the cognition layer, the choice depends on what the network goals are and how these problems are set up. Lastly, the feedback loop is completed with the input from the network, physical resources and users.

Clark et al. proposed in [39] for the first time, the idea of a new network that could assemble itself, reassemble itself as conditions change, automatically detect problems in the network and fix them if possible with the use of a knowledge-based control plane. The requirements of an effective knowledge-based control plane are defined in [39] as follows.

- It should be distributed.

- It should be bottom up (simple entities can compose complex ones).

- It should be constraint driven.

- Moves from simple to complex in the sense that composition of simple entities creates more requirements or constraints.

- It must be able to collect, filter reduce and distribute observations, assertions and extract conclusions from different parts of the network.

- It must operate successfully with incomplete and inaccurate information.

- It must be prepared to reason about the collected information.

Based on the proposed description of the knowledge plane, the authors in [40] proposed an architecture based on machine learning tools, which could be integrated in autonomic 
architectures instead of considering it as a new autonomic architecture itself. Cognitive networking as defined in [11] introduces intelligence into the network architectures. Network becomes an active, intelligent and autonomous element able to interact with and adapt to the environment.

Another architecture proposal is CogNet [41], which gathers information in a distributed environment and also stores this information again in a distributed repository. The aim is to combine the experience of each node in the network and use it to leverage the overall network performance. The authors present their architecture's performance with CogTCP, the CogNet enabled TCP. In a CogTCP enabled wireless access point, the experiences from the same network to the same destination are accumulated. Then, in a multiple ongoing connection scenario, the end nodes fetch the accumulated information from the distributed repository and make use of them to leverage their TCP performance such as avoiding the slow start phase and unnecessary throughput degradation related to congestion control.

A cognitive Resource Manager (CRM) is proposed in [42] where the knowledge-plane is employed. The CRM has a machine learning toolbox which carries out cross layer optimizations applied on a great variety of information from the application layer, the underlying networking and data link layer as well as the operating system. The toolbox has a wide spectrum involving artificial neural networks, Bayesian reasoning, genetic algorithms, time series analysis, simulated annealing and coloring.

A channel allocation architecture depending on the island genetic algorithm is presented in [43]. While using the island genetic algorithm for distributed optimization, a fuzzy logic controller is being considered to reduce the uncertainty and noise at the input.

SmartA [44] is a collection of previously proposed architectures on cognitive networks. It integrates Context Manager [45], Resource Manager [46], Stack Manager [42] and introduces Service Manager. The Service Manager's role is to maximize the user satisfaction by fulfilling the user requirements as best as possible.

\subsection{Cognition in CCNP}

Present networking and resource utilization functions follow the layered protocol architecture. By design, the abstracted layers have little information regarding the other layers. Because of this unawareness, the network and resource utility maximization problems being solved today produce sub-optimal results. An integrated scheme that imitates the human approach may produce better results. Therefore, we define the role of the cognitive control plane in the CCNP architecture as replacing the network and resource manager as much as possible. Within this scope, the objective of the cognitive control plane is to observe, act and learn to produce better results in real-time. This scheme is being addressed in cognitive radio networks, which, in principle, operates in the physical and data-link layer of the OSI stack and is usually unaware of the needs of the other layers and users. Beyond the network control functionality, the aim of introducing cognition in the resource life cycle is to understand and anticipate the user needs. To achieve this objective, context awareness is required to enable the decision making process which imposes the situatedness challenge on the cognitive control plane. Situatedness challenge may be described as being embedded in the environment and infrastructure to monitor the states. In this approach, the cognitive control plane has to have enough degrees of freedom in acting [47].

In essence, two approaches can be employed to introduce intelligent control in the architecture, i.e.: centralized cognitive control entity or distributed middleware that runs on each device as a virtualization layer. A hybrid approach in which distributed and centralized 
functionalities merge may perform better. In the centralized implementation, there is a central entity in the network to run the cognitive functionalities. In the distributed middleware approach, each entity in the network runs their own cognitive functionalities with or without communicating to each other. In the distributed scheme, cognition becomes ubiquitous. If the centralized approach is employed, defining only the individual cognitive control plane is not enough to have a complete view of the system. The emergent behavior, which is a property of a collection of subsystems due to their interactions, of the cognitive planes must also be analyzed. For example, the cognitive control plane implemented on a device may exhibit some selfish behavior. The overall impact of this selfishness may be balanced by adequate selection of the strategies in the community of the devices. Such circumstances require collaboration among the cognitive elements. An example is presented in Sect. 3 about malicious or selfish nodes in an ad hoc network.

The cognitive engine in the cognitive control plane solves multi-objective optimization problems. Therefore, the solutions are Pareto-optimal [11]. Based on the gathered context, the cognitive engine takes decisions. The elements in the cognitive engine may be neural networks, swarm intelligence, evolutionary programming, immune or fuzzy systems, etc. The cognitive engine should adapt itself continuously using the feedbacks. The communication stack provides feedbacks to the cognitive control plane as shown in Fig. 5. The cognitive application programming interface (CAPI) provides the required interface between the virtualized resources and the cognitive control plane [47].

The cognitive control plane interacts with the users and application layer as shown in Fig. 7 to achieve three basic objectives:

1. collecting information from the users and applications through direct or indirect observations, or service level negotiations to learn and anticipate the user needs,

2. allowing the user to be in the control loop of the cognitive decision process. Although, for the sake of autonomous operation the second functionality should be minimal, from time to time user interventions may be required.

3. to give feedback to the user.

The cognitive control plane requires input through observations of the states of the virtual or physical resources via sensors; e.g., state of the congestion levels in the network, to which the multimedia trunks are assigned, residual battery levels, queue sizes of the services, memory utilization of the applications or user behaviors. In order to learn the behavior of the virtual infrastructure, context information must be gathered and stored in a database. Instead of using the vast amount of unprocessed data, the input can be filtered to extract the useful information that can be considered as feature extraction. Accuracy, timeliness and the price of the ignorance are the key measures to be analyzed [11]. The price of ignorance is the impact when a piece of data which is relevant to the decision is filtered out.

The research challenges of introducing intelligent control over the resources in the CCNP can be summarized as follows:

- A cognitive control plane must be developed to learn and control the network elements, aggregations and relationships among network elements, to keep track of transport entities, and their endpoints, to monitor alarms, to configure resources. How to introduce cognition into the several layers of the heterogeneous systems to satisfy the system and user objectives imposed by the transsectoral applications is a major challenge. The implementation may be centralized, distributed or a combination of these two schemes.

- Decisions produced by the cognitive control plane are generally required in real-time. Therefore, the elements of the cognitive control plane are to be designed to support real-time resource management. 


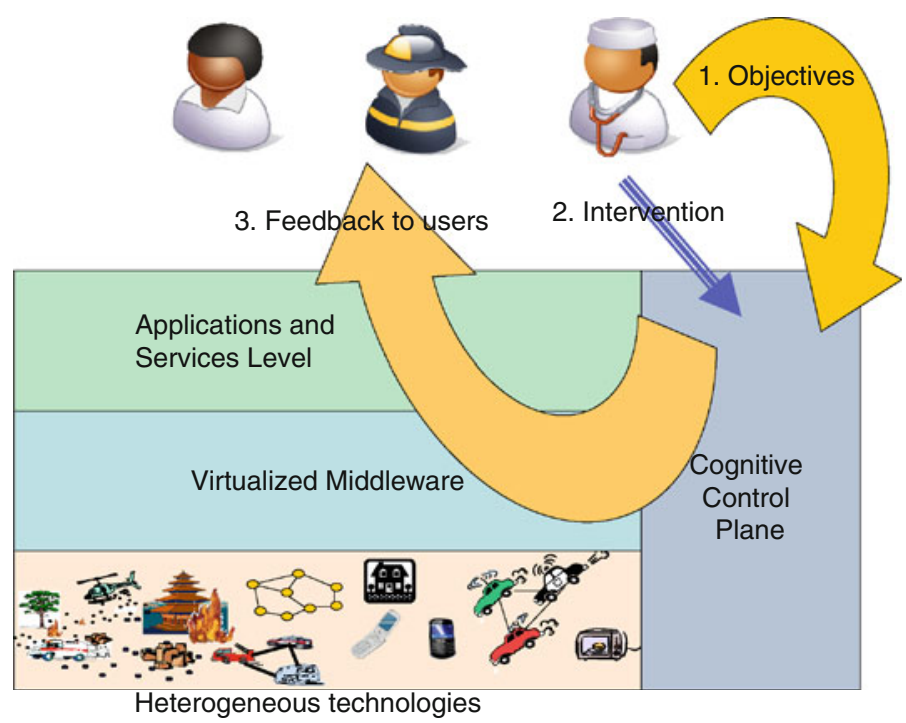

Fig. 7 The cognitive control plane interacts with the users and application layer to achieve three basic objectives

- The scene analysis and situatedness challenge must be studied. In cognitive science, situatedness is being embedded in the environment and infrastructure to monitor the environment and the states of the architectural elements. In this scope, sensor networking applications may explicitly collaborate with the CCNP or may be within the CCNP design.

- The cognitive network application programming interface must be specified. Definition of the objectives, the management primitives, feedback loops, resource mappings are part of the application programming interface.

- An intelligent resource mapping mechanism must be developed. When the heterogeneity of the underlying devices and networks are considered, there will be a large number of resources. These resources must be mapped to virtual resources to enable portability of applications and enhancement of the resources without affecting the already developed applications. Therefore, one of the research challenges is mapping of the resources to corresponding virtual resources. This sort of "mapping" must provide enough information about the capabilities of the resources, while the device/network capability information must be harmonized by virtualization.

- The price of control is also to be analyzed [11]. Introducing the cognitive plane and the required messaging to bring in intelligence in the virtualized infrastructure may use in-band communication resources; therefore, the trade-off between the overhead and benefits must be analyzed.

- Developing mechanisms to make the virtualization layer as smart as possible to organize and heal itself to reduce the number of user interventions. From the viewpoint of the user, this reduces the complexity and increases the manageability.

- The devices and users are mobile. Therefore, the opportunistic and dynamic nature of the underlying wireless communication technologies must also be exploited in the CCNP. This should be done to handle the dynamic nature that can be expected from the underlying networks. 
- Along with the four logical layers of network management defined by the International Telecommunication Union (business, service, network and element), the user management must be incorporated into the cognitive control plane to manage the user requirements and to give feedback to the user.

- Link-level cognitive architecture and cooperation mechanisms (protocols, interfaces, algorithms) must be studied to enable the cognitive control plane of the CCNP.

- The co-existence issues among collaborating technologies must be analyzed. Transmission adaptation, dynamic frequency selection algorithms must be revisited to overcome the co-existence problems.

- The naming, addressing and identity management issues must be studied in the CCNP domain. There may be multiple trust domains within the CCNP.

- Horizontal, vertical and multiple-stack collaboration techniques must be studied considering the communication, operational and social cooperation levels.

\section{Conclusions}

With the recent proliferation of devices and networking technologies, the control and management of the networked applications will become very complex. In this paper, we present the opportunities and challenges of a visionary concept referred to as collaborative and cognitive networked platforms. The collaborative and cognitive network platforms may be an adequate architecture to solve the scalability issues of control and management. We presented the architecture in two major dimensions: cognition and collaboration. Cognition is required to replace the operations manager and to make the system better adaptive to dynamics in the system. Collaboration is studied to enhance the quality of service or experience provided by the system by creating a holistic architecture where heterogeneous entities strive to achieve a common goal in a dynamic and opportunistic fashion. The research challenges pertinent to these two domains are also presented.

Open Access This article is distributed under the terms of the Creative Commons Attribution Noncommercial License which permits any noncommercial use, distribution, and reproduction in any medium, provided the original author(s) and source are credited.

\section{References}

1. Onur, E., Deliç, H., Ersoy, C., \& Caglayan, M. (2002). On the retrial and redial phenomena in GSM networks. In IEEE wireless communications and networking conference (Vol. 2, pp. 885-889).

2. Bur, K., \& Ersoy, C. (2005). Ad hoc quality of service multicast routing. Computer Communications, 29(1), 136-148.

3. Jefferies, N. (2007). Global vision for a wireless world. In 18th wireless world research forum meeting, Helsinki, Finland.

4. Jacobsson, M., Niemegeers, I., \& de Groot, S. H. (2010). Personal networks: Wireless networking for personal devices. New York: Wiley.

5. Fortuna, C., \& Mohorcic, M. (2009). Trends in the development of communication networks: Cognitive networks. Computer Networks, 53(9), 1354-1376.

6. Onur, E., Jacobsson, M., de Groot, S. H., \& Niemegeers, I. (2008). Manageable bubbles of the future Internet: Personal virtual super devices. In Wireless World Research Forum.

7. Onur, E., Ersoy, C., \& Delic, H. (2005). Quality of deployment in surveillance wireless sensor networks. International Journal of Wireless Information Networks, 12(1), 61-67.

8. Rainie, L., Fox, S., \& Anderson, J. (2005). The future of the Internet.

9. Tu, Y. (2000). How robust is the Internet? Nature, 406, 353-354. 
10. Sagarin, R. D., Alcorta, C. S., Atran, S., Blumstein, D. T., Dietl, G. P., Hochberg, M. E., et al. (2010). Decentralize, adapt and cooperate. Nature, 465, 292-293.

11. Thomas, R. W., Friend, D. H., DaSilva, L. A., \& MacKenzie, A. B. (2006). Cognitive networks: Adaptation and learning to achieve end-to-end performance objectives. IEEE Communications Magazine, 44(12), 51-57.

12. Ohtsuki, H., Hauert, C., Lieberman, E., \& Nowak, M. A. (2006). A simple rule for the evolution of cooperation on graphs and social networks. Nature, 441, 502-505.

13. Keller, L., \& Reeve, H. K. (1998). Familiarity breeds cooperation. Nature, 394, 121-122.

14. Taylor, P. D., \& Day, T. (2004). Behavioural evolution: Cooperate with thy neighbour? Nature, 428, 611-612.

15. Mesterton-Gibbons, M. (2010). Why fairness pays. Nature, 464, 1280.

16. IETF, Problem statement for cross-layer optimization. Internet Draft, Status: Informational, Expires: January 2011, July 12, 2010. [Online]. Available: http://tools.ietf.org/html/draft-lee-cross-layeroptimization-problem-01.

17. Zhou, J., Jacobsson, M., Onur, E., \& Niemegeers, I. (2010). An investigation of link quality assessment for mobile multi-hop and multi-rate wireless networks. Wireless Personal Communication. doi: 10.1007/s11277-011-0263-1.

18. Kwon, T., Lee, H., Choi, S., Kim, J., Cho, D.-H., et al. (2005). Design and implementation of a simulator based on a cross-layer protocol between mac and phy layers in a wibro compatible. IEEE 802.16e OFDMA system. IEEE Communication Magazine, 43(12), 136-146.

19. Clausen, T., Jacquet, P., Adjih, C., Laouiti, A., Minet, P., Muhlethaler, P., et al. (2003). Optimized link state routing protocol OLSR. IETF RFC.

20. Shakkottai, S., Rappaport, T., \& Karlsson, P. (2003). Cross-layer design for wireless networks. IEEE Communications Magazine, 41(10), 74-80.

21. Demirkol, I., Ersoy, C., \& Onur, E. (2009). Wake-up receivers for wireless sensor networks: Benefits and challenges. Wireless Communications, IEEE, 16(4), 88-96.

22. Genç, Z., Rizvi, U. H., Onur, E., \& Niemegeers, I. (2010). Cooperative communications in future home networks. Wireless Personal Communication, 53(3), 349-364.

23. Moh, S., \& Yu, C. (2010). A cooperative diversity-based robust mac protocol in wireless ad hoc networks. IEEE Transactions on Parallel and Distributed Systems, 99, 1.

24. Park, T., Kim, M., Kim, C., \& Jung, J. (2010). A cooperative communication system using cross-layer coding method base on hybrid-arq. Second international conference on ubiquitous and future networks (ICUFN), pp. 145-149.

25. Genc, Z., Olcer, G. M., Onur, E., \& Niemegeers, I. (2010). Improving 60 ghz indoor connectivity with relaying. In Proceedings of IEEE international communications conference (ICC), pp. 1-6.

26. Singh, S., Ziliotto, F., Madhow, U., Belding, E., \& Rodwell, M. (2009). Blockage and directivity in $60 \mathrm{GHz}$ wireless personal area networks: From cross-layer model to multihop mac design. IEEE Journal on Selected Areas in Communications, 27(8), 1400-1413.

27. Fan, Z. (2008). Wireless networking with directional antennas for $60 \mathrm{GHz}$ systems. In Proceedings of 14th European wireless conference (EW), pp. 1-7.

28. http://www.nas.ewi.tudelft.nl/research/triangle/index.html.

29. Chandrasekhar, V., Andrews, J., \& Gatherer, A. (2008). Femtocell networks: A survey. IEEE Communications Magazine, 46(9), 59-67.

30. Claussen, H., Ho, L. T. W., \& Samuel, L. G. (2008). An overview of the femtocell concept. Bell Labs Technical Journal, 13(1), 221-245.

31. [Online]. Available: http://www.femtoforum.org.

32. Marron, P., Karnouskos, S., Minder, D., et al. (2009). Research roadmap on cooperating objects. The CONET Consortium.

33. Lin, E.-Y., Rabaey, J., \& Wolisz, A. (2004). Power-efficient rendez-vous schemes for dense wireless sensor networks (Vol. 7, pp. 3769-3776).

34. Onur, E., Ersoy, C., Delic, H., \& Akarun, L. (2006). Coverage in sensor networks when obstacles are present. IEEE International Conference on Communications, 9, 4077-4082.

35. Neisser, U. (1967). Cognitive psychology. New York: Appleton-Century-Crofts.

36. Mitola, J., et al. (2000). Cognitive radio: an integrated agent architecture for software defined radio. Ph.D. dissertation.

37. Best, J. (1986). Cognitive psychology. New York: West Publishing Co.

38. Thomas, R., DaSilva, L., \& MacKenzie, A. (2005). Cognitive networks. In First IEEE international symposium on new frontiers in dynamic spectrum access networks, 2005. DySPAN 2005, pp. 352-360. 
39. Clark, D. D., Partridge, C., Ramming, J. C., \& Wroclawski, J. T. (2003). A knowledge plane for the internet. In Proceedings of the 2003 conference on applications, technologies, architectures, and protocols for computer communications. ACM, New York, NY, USA, pp. 3-10.

40. Mbaye, M., \& Krief, F. (2009). A collaborative knowledge plane for autonomic networks. In Autonomic communication.Springer, New York, US, pp. 69-92.

41. Manoj, B., Rao, R., \& Zorzi, M. (2008). CogNet: A cognitive complete knowledge network system. IEEE Wireless Communications, 15(6), 81-88.

42. Mähönen, P., Petrova, M., Riihijärvi, J., \& Wellens, M. (2006). Cognitive wireless networks: Your network just became a teenager. In Proceedings of IEEE INFOCOM 2006.

43. Friend, D., EINainay, M., Shi, Y., \& MacKenzie, A. (2008). Architecture and performance of an island genetic algorithm-based cognitive network. In Consumer communications and networking conference, 2008. CCNC 2008. 5th IEEE, pp. 993-997.

44. Fortuna, C., \& Mohorcic, M. (2008). Advanced access architecture for efficient service delivery in heterogeneous wireless networks. In Third international conference on communications and networking in China, 2008. ChinaCom 2008, pp. 1173-1177.

45. Strassner, J. (2007). In Q. H. Mahmoud (Ed.), The role of autonomic networking in cognitive networks. Wiley, New York.

46. Sutton, P., Doyle, L. E., \& Nolan, K. E. (2006). A reconfigurable platform for cognitive networks. In 1 st International conference on cognitive radio oriented wireless networks and communications, pp. 1-5.

47. Onur, E., Sfakianakis, E., Papagianni, C., Karagiannis, G., Kontos, T., Niemegeers, I. et al. (2009). Intelligent end-to-end resource virtualization using service oriented architecture. In Proceedings of IEEE GLOBECOM workshops, pp. 1-6.

\section{Author Biographies}

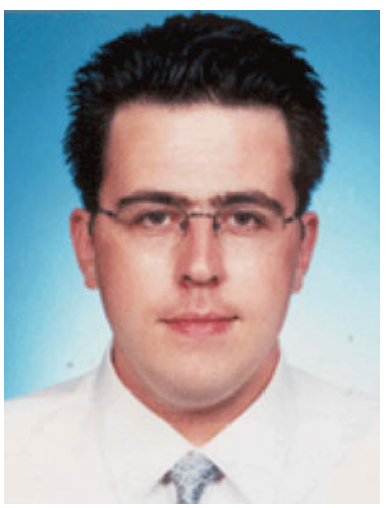

Ertan Onur received the B.Sc. degree in computer engineering from Ege University, Izmir, Turkey in 1997, and the M.Sc. and Ph.D. degrees in computer engineering from Bogazici University, Istanbul, Turkey in 2001 and 2007, respectively. After the B.Sc. degree, he worked for LMS Durability Technologies GmbH, Kaiserslautern, Germany. During the M.Sc. and Ph.D. degrees, he worked as a project leader at Global Bilgi, Istanbul and as an R\&D project manager at Argela Technologies, Istanbul. He developed and managed many commercial telecommunications applications, has a patent and published more than forty papers. Presently, he is an assistant professor at EEMCS, WMC, Delft University of Technology, the Netherlands. He is the editor/convenor of the Personal Networks Group of Ecma International Standardization Body. Dr. Onur's research interests are in the area of cooperative networks, personal networks, wireless and sensor networks. He is a member of IEEE.

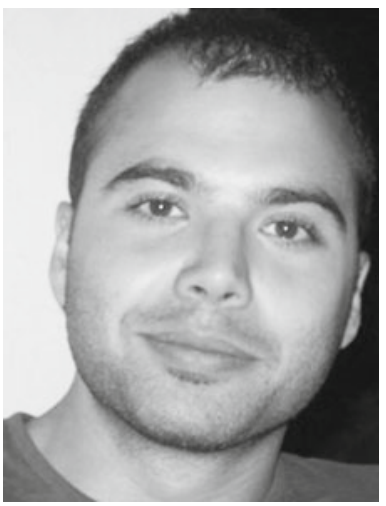

Yunus Durmus received his B.Sc. and M.Sc. degrees in computer engineering from Bogazici University, Istanbul, Turkey in 2007 and 2009 , respectively. He is currently pursuing for the Ph.D. degree in EEMCS department at Wireless and Mobile Communications Group at Delft University of Technology. His research interests include cognitive and cooperative networks, performance analysis and web technologies. 


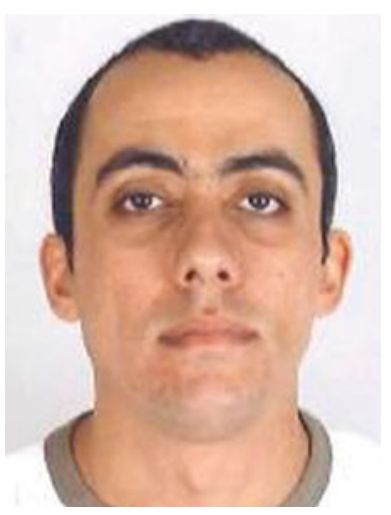

Mohamed Gamal Hawas received the B.Sc. degree in Electrical Engineering from Delft University of Technology, Delft, Netherlands in 2007. In 2009 he received the M.Sc. degree in Mobile and Wireless Communications at the same university. He started Ph.D. research at Delft University (WMC group). His research interests include ICT for smart public services, wireless communications and cooperative networking.

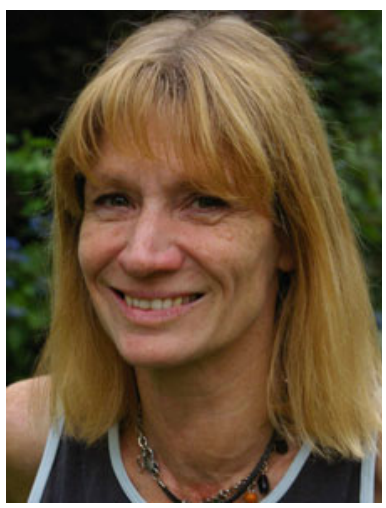

Sonia Marcela Heemstra de Groot received a Ph.D. degree in Electrical Engineering from the University of Twente, The Netherlands, in 1990. Since 2003 she holds the position of chief scientist at WMC, of which she is one of the co-founders. She is full-professor at Delft University of Technology, where she holds the part-time chair in Personal and Ambient Networking. She has authored or co-authored more than 100 papers published in international journals or presented at international conferences. She has participated in many national and international projects.

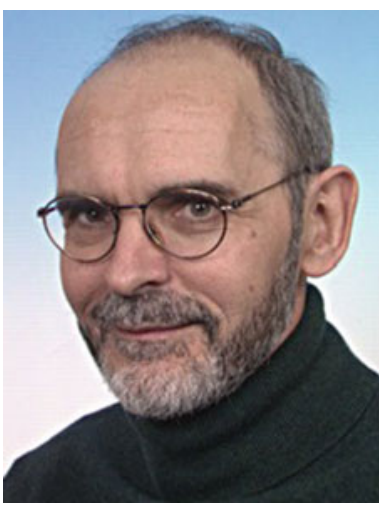

Ignas G. M. M. Niemegeers got a degree in Electrical Engineering from the University of Gent, Belgium, in 1970. In 1972 he received a M.Sc.E. degree in Computer Engineering and in 1978 a Ph.D. degree from Purdue University in West Lafayette, Indiana, USA. From 1978 to 1981 he was a designer of packet switching networks at Bell Telephone Mfg. Cy, Antwerp, Belgium. From 1981 to 2002 he was a professor at the Computer Science and the Electrical Engineering Faculties of the University of Twente, Enschede, The Netherlands. From 1995 to 2001 he was Scientific Director of the Centre for Telematics and Information Technology (CTIT) of the University of Twente, a multi-disciplinary research institute on ICT and applications. Since May 2002 he holds the chair Wireless and Mobile Communications at Delft University of Technology, where he is heading the Centre for Wireless and Personal Communication (CWPC) and the Telecommunications Department. He was involved in many European research projects, e.g., the EU projects MAGNET and MAGNET Beyond on personal networks, EUROPCOM on UWB emergency networks and, eSENSE and CRUISE on sensor networks. He is a member of the Expert group of the European technology platform eMobility and IFIP TC-6 on Networking. His present research interests are $4 \mathrm{G}$ wireless infrastructures, future home networks, ad-hoc networks, personal networks, cognitive networks. 